\title{
SPHERICAL SYMMETRY OF SOME UNITARY INVARIANTS FOR COMMUTING TUPLES
}

\author{
SAMEER Chavan AND KaIs FeKi
}

\begin{abstract}
We discuss spherical and Euclidean analogues of joint spectral radius, joint operator norm and joint numerical radius associated with commuting $d$-tuples of Hilbert space operators. In particular, we deduce their invariance under the action of the group $\mathscr{U}(d)$ of $d \times d$ unitary matrices. Unlike spectral and numerical radii, the analogues of joint operator norm differ in dimension $d>1$. The joint hyponormality ensures that these analogues of joint operator norm agree in all dimensions. However, the separate hyponormality fails to ensure so.
\end{abstract}

Mathematics subject classification (2020): 47A13, 47B20.

Keywords and phrases: Unitary invariants, Taylor spectrum, joint hyponormality.

\section{REFERENCES}

[1] W. Arveson, Subalgebras of $C^{*}$-algebras, III. Multivariable operator theory, Acta Math. 181 (1998), 159-228.

[2] A. Athavale, On joint hyponormality of operators, Proc. Amer. Math. Soc. 103 (1988), 417-423.

[3] H. BAKLOUTI, K. FeKI, On joint spectral radius of commuting operators in Hilbert spaces, Linear Algebra Appl. 557 (2018) 455-463.

[4] H. Baklouti, K. Feki, O. A. M. Sid Ahmed, Joint numerical ranges of operators in semiHilbertian spaces, Linear Algebra Appl. 555 (2018) 266-284.

[5] J. W. BUnCE, Models for n-tuples of noncommuting operators, J. Funct. Anal. 57 (1984), 21-30.

[6] S. Chavan, V. M. Sholapurkar, Completely hyperexpansive tuples of finite order, J. Math. Anal. Appl. 447 (2017) 1009-1026.

[7] S. Chavan, D. Yakubovich, Spherical tuples of Hilbert space operators, Indiana Univ. Math. J. 64 (2015), 577-612.

[8] M. Chō, M. TAKaguchi, Boundary points of joint numerical ranges, Pacific journal of math 95, (1981), 27-35.

[9] M. Chō, W. ŻELAZKo, On geometric spectral radius of commuting $n$-tuples of operators, Hokkaido Math. J., 21 (1992), 251-258.

[10] R. E. CURTO, Applications of several complex variables to multiparameter spectral theory. In Surveys of some recent results in operator theory, Vol. II, volume 192 of Pitman Res. Notes Math. Ser., pages 25-90. Longman Sci. Tech., Harlow, 1988.

[11] R. E. CURTO, Joint hyponormality: a bridge between hyponormality and subnormality, Operator theory: operator algebras and applications, Part 2 (Durham, NH, 1988), 69-91, Proc. Sympos. Pure Math., 51, Part 2, Amer. Math. Soc., Providence, RI, 1990.

[12] C. GU, Examples of m-isometric tuples of operators on a Hilbert space, J. Korean Math. Soc. 55 (2018), 225-251.

[13] K. FEKI, A note on doubly commuting tuples of hyponormal operators on Hilbert spaces, Results Math 75, 93 (2020), https://doi.org/10.1007/s00025-020-01220-5.

[14] P. R. Halmos, A Hilbert space problem book, Vol. 19, Springer Science and Business Media, 2012.

[15] V. MÜLLER, A. SOLTYSIAK, Spectral radius formula for commuting Hilbert space operators, Studia Math. 103 (1992), 329-333.

[16] G. Popescu, Unitary invariants in multivariable operator theory, Memoirs of the American Mathematical Society, 200 (2009), vi+91 pp. 
[17] J. L. TAYLOR, A joint spectrum for several commuting operators, J. Funct. Anal. 6 (1970) 172-191. 